\title{
NDRG1 Expression Is an Independent Prognostic Factor in Inflammatory Breast Cancer
}

\author{
Emilly S. Villodre ${ }^{1,2}{ }^{-}$, Yun Gong ${ }^{2,3}$, Xiaoding Hu ${ }^{1,2}$, Lei Huo ${ }^{2,3}$, Esther C. Yoon ${ }^{3}{ }^{(D}$, \\ Naoto T. Ueno ${ }^{1,2} \mathbb{D}$, Wendy A. Woodward ${ }^{2,4}$, Debu Tripathy ${ }^{1} \mathbb{D}$, Juhee Song ${ }^{5}$ \\ and Bisrat G. Debeb 1,2,* \\ 1 Department of Breast Medical Oncology, The University of Texas MD Anderson Cancer Center, \\ Houston, TX 77030, USA; esschlee@mdanderson.org (E.S.V.); xhu7@mdanderson.org (X.H.); \\ nueno@mdanderson.org (N.T.U.); dtripathy@mdanderson.org (D.T.) \\ 2 Morgan Welch Inflammatory Breast Cancer Clinic and Research Program, The University of Texas \\ MD Anderson Cancer Center, Houston, TX 77030, USA; yungong@mdanderson.org (Y.G.); \\ leihuo@mdanderson.org (L.H.); wwoodward@mdanderson.org (W.A.W.) \\ 3 Department of Pathology, The University of Texas MD Anderson Cancer Center, Houston, TX 77030, USA; \\ ecyoon@mdanderson.org \\ 4 Department of Radiation Oncology, The University of Texas MD Anderson Cancer Center, \\ Houston, TX 77030, USA \\ 5 Department of Biostatistics, The University of Texas MD Anderson Cancer Center, Houston, TX 77030, USA; \\ jsong1@mdanderson.org \\ * Correspondence: bgdebeb@mdanderson.org; Tel.: +1-713-792-0696
}

Received: 3 November 2020; Accepted: 8 December 2020; Published: 10 December 2020

Simple Summary: Inflammatory breast cancer (IBC) is a rare and aggressive variant of breast cancer that is responsible for a significant number of breast cancer-related deaths. Herein, we describe how the expression of a specific protein named N-myc downstream-regulated gene 1 (NDRG1), commonly described as a gene that prevents the spread of cancer cells to distant organs, may have a paradoxical role in cancer progression in IBC. We found that the level of expression of NDRG1 in tumor tissues predicts the survival outcome of patients with IBC. We also observed that NDRG1, together with other important prognostic factors such as estrogen receptor status and stage, could be used to further analyze prognostic outcome or treatment response of patients.

Abstract: NDRG1 is widely described as a metastasis suppressor in breast cancer. However, we found that NDRG1 is critical in promoting tumorigenesis and brain metastasis in mouse models of inflammatory breast cancer (IBC), a rare but highly aggressive form of breast cancer. We hypothesized that NDRG1 is a prognostic marker associated with poor outcome in patients with IBC. NDRG1 levels in tissue microarrays from 64 IBC patients were evaluated by immunohistochemical staining with NDRG1 (32 NDRG1-low ( $\leq$ median), 32 NDRG1-high (>median)). Overall and disease-free survival (OS and DSS) were analyzed with Kaplan-Meier curves and log-rank test. Univariate analysis showed NDRG1 expression, tumor grade, disease stage, estrogen receptor (ER) status, and receipt of adjuvant radiation to be associated with OS and DSS. NDRG1-high patients had poorer 10-year OS and DSS than NDRG1-low patients (OS, $19 \%$ vs. $45 \%, p=0.0278$; DSS, $22 \%$ vs. $52 \%, p=0.0139$ ). On multivariable analysis, NDRG1 independently predicted OS (hazard ratio $(\mathrm{HR})=2.034, p=0.0274$ ) and DSS $(\mathrm{HR}=2.287, p=0.0174)$. NDRG1-high ER-negative tumors had worse outcomes OS, $p=0.0003$; DSS, $p=0.0003$; and NDRG1-high tumors that received adjuvant radiation treatment had poor outcomes (OS, $p=0.0088$; DSS, $p=0.0093$ ). NDRG1 was a significant independent prognostic factor for OS and DSS in IBC patients. Targeting NDRG1 may represent a novel strategy for improving clinical outcomes for patients with IBC. 
Keywords: NDRG1; N-myc downstream-regulated gene 1; IBC; inflammatory breast cancer; survival

\section{Introduction}

Inflammatory breast cancer (IBC) is one of the most aggressive forms of breast cancer. Although rare, accounting for only $1 \%-4 \%$ of newly diagnosed breast cancer cases, it is responsible for a disproportionately high $10 \%$ of breast cancer-related deaths in the United States [1,2]. IBC has a unique biology characterized by rapid proliferation and metastasis; indeed, almost all patients have lymph node involvement and more than $33 \%$ of patients with IBC present with distant metastasis at the time of diagnosis [3,4]. Even with multimodality treatment approaches that include systemic chemotherapy, surgery, and radiation therapy, the prognosis for patients with IBC is worse than for non-IBC patients (overall survival (OS) rates $40 \%$ versus $63 \%$ at 5 years) [5-7]. This may be due in part to $70 \%$ of IBC patients presenting with aggressive subtypes of HER2+ or triple-negative breast cancer (TNBC), compared with $40 \%$ of non-IBC tumors [8]. Efforts have been undertaken to identify molecular markers and therapeutic targets distinct to IBC and have identified important targets and pathways, including EGFR, E-cadherin, eIFG4I, RhoC, and TIG1/AXL [9-13]. However, no IBC-specific molecular signature or target has been identified thus far, and effective targeted therapies for this disease remain limited.

$\mathrm{N}$-myc downstream-regulated gene 1 (NDRG1) is a stress response protein involved in hypoxia, cell growth, lipid metabolism, and resistance to chemotherapy [14-19]. NDRG1 is widely known as a metastasis suppressor in breast cancer, acting mainly by suppressing migration and invasion of breast cancer cells [20-22]. However, we and others have shown NDRG1 to be a tumor promoter in aggressive breast cancer [23-25]. Nagai and colleagues also showed that high expression of NDRG1 was associated with aggressive breast cancer behaviors, including the advanced stage at presentation and high-grade tumors and that NDRG1 was independently associated with poor survival outcome [26]. However, the expression of NDRG1 and its clinical importance in IBC remains unknown.

Herein, we examined the expression of NDRG1 by using immunohistochemical staining of a tissue microarray (TMA) composed of samples from IBC patients and evaluated the expression of NDRG1 and its correlation with survival outcomes. We also assessed the association between NDRG1 expression and outcome stratified by known prognostic factors. Our findings showed that high expression of NDRG1 in IBC tumors was an independent predictor of worse OS and disease-specific survival (DSS).

\section{Results}

To determine whether NDRG1 protein expression is associated with outcome in IBC, immunohistochemical staining was performed on TMAs from 64 patients with primary IBC who were treated between 1991 and 2004 at The University of Texas MD Anderson Cancer Center. The tissues used to create the TMA were from refractory or residual IBC tumors after neoadjuvant systemic therapy. The average age of these patients was 50 years (range 23-75 years). Eighty-three percent of patients were stage III, $80 \%$ high grade, $62 \%$ were ER-negative tumors, and $67 \%$ of these patients received adjuvant radiation. The median follow-up time for the patients studied was 11.7 years, and the median OS time was 3.7 years. NDRG1 staining was predominantly cytoplasmic/membranous. Representative images of NDRG1-low and -high tumors are shown in Figure 1. 
a

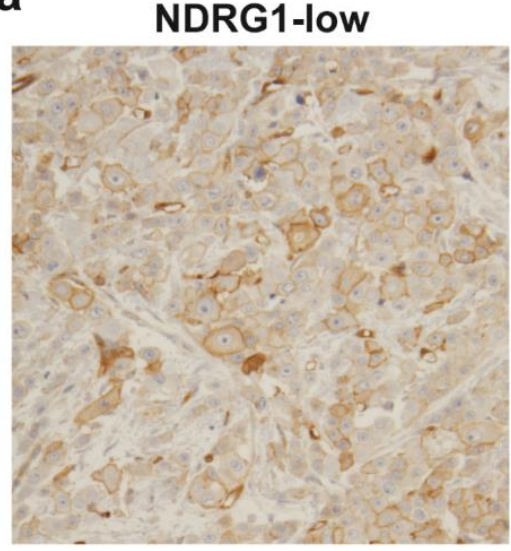

b

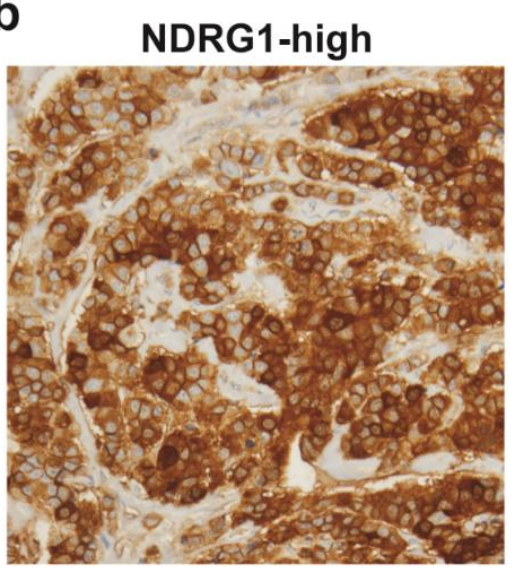

Figure 1. Immunohistochemical staining of N-myc downstream-regulated gene 1 (NDRG1) in inflammatory breast cancer (IBC) tumors. Representative images of NDRG1 immunostaining of (a) an NDRG1-low IBC tumor and (b) an NDRG1-high IBC tumor.

Table 1 summarizes patient characteristics based on NDRG1 expression status, NDRG1 expression was associated with negative HER2 status $(p=0.0077)$. Univariate analysis (Table 2$)$ showed that NDRG1 expression (hazard ratio $(\mathrm{HR})=2.1, p=0.0150)$, tumor grade $(\mathrm{HR}=2.4, p=0.0463)$, disease stage ( $\mathrm{HR}=4.6, p=0.0011)$, ER status ( $\mathrm{HR}=0.4, p=0.0098)$, and adjuvant radiation therapy $(\mathrm{HR}=0.5$, $p=0.0434)$ were associated with OS. The same variables were also associated with DSS (Table 2).

Table 1. Clinical and pathologic characteristics of tumor samples from patients with IBC according to NDRG1 expression.

\begin{tabular}{|c|c|c|c|c|}
\hline Covariate & Level & $\begin{array}{l}\text { NDRG1-Low } \\
\quad(n=32)\end{array}$ & $\begin{array}{l}\text { NDRG1-High } \\
\quad(n=32)\end{array}$ & $p$-Value \\
\hline Age & & $51.5 \pm 12.1$ & $48.6 \pm 12$ & 0.3861 \\
\hline \multirow[t]{2}{*}{ Race } & Non-white & $8(25 \%)$ & $5(16 \%)$ & 0.5356 \\
\hline & White & $24(75 \%)$ & $27(84 \%)$ & \\
\hline \multirow[t]{2}{*}{ Histologic type } & Others & $2(6 \%)$ & $4(13 \%)$ & 0.6719 \\
\hline & Ductal & $30(94 \%)$ & $28(87 \%)$ & \\
\hline \multirow[t]{2}{*}{ Grade } & $1-2$ & $6(19 \%)$ & $7(22 \%)$ & 0.7560 \\
\hline & 3 & $26(81 \%)$ & $25(78 \%)$ & \\
\hline \multirow{2}{*}{$\begin{array}{l}\text { Lymphovascular } \\
\text { invasion }\end{array}$} & No & $4(14 \%)$ & $3(10 \%)$ & 0.7065 \\
\hline & Yes & $25(86 \%)$ & $27(90 \%)$ & \\
\hline \multirow[t]{2}{*}{ Stage } & III & $31(97 \%)$ & $27(84 \%)$ & 0.1961 \\
\hline & IV & $1(3 \%)$ & $5(16 \%)$ & \\
\hline \multirow[t]{2}{*}{ Estrogen receptor } & No & $21(66 \%)$ & $19(61 \%)$ & 0.7209 \\
\hline & Yes & $11(34 \%)$ & $12(39 \%)$ & \\
\hline \multirow[t]{2}{*}{$\begin{array}{l}\text { Progesterone } \\
\text { receptor }\end{array}$} & No & $24(75 \%)$ & $19(61 \%)$ & 0.2425 \\
\hline & Yes & $8(25 \%)$ & $12(39 \%)$ & \\
\hline \multirow[t]{2}{*}{ HER2 } & No & $12(37 \%)$ & $22(71 \%)$ & 0.0077 \\
\hline & Yes & $20(63 \%)$ & $9(29 \%)$ & \\
\hline \multirow[t]{2}{*}{$\begin{array}{l}\text { Triple-negative } \\
\text { breast cancer }\end{array}$} & No & $27(84 \%)$ & $20(64 \%)$ & 0.0879 \\
\hline & Yes & $5(16 \%)$ & $11(36 \%)$ & \\
\hline \multirow[t]{2}{*}{ Adjuvant radiation } & No & $10(31 \%)$ & $11(34 \%)$ & 0.7901 \\
\hline & Yes & $22(69 \%)$ & $21(66 \%)$ & \\
\hline
\end{tabular}


Table 2. Univariate Cox regression analysis on overall survival and disease-specific survival among patients with IBC.

\begin{tabular}{|c|c|c|c|c|c|c|c|}
\hline \multirow[b]{2}{*}{ Covariate } & \multirow[b]{2}{*}{ Level } & \multicolumn{3}{|c|}{ Overall Survival } & \multicolumn{3}{|c|}{ Disease-Specific Survival } \\
\hline & & HR & $95 \%$ CI & $p$-Value & HR & $95 \% \mathrm{CI}$ & $p$-Value \\
\hline \multirow{3}{*}{$\begin{array}{c}\text { Age } \\
\text { NDRG1 }\end{array}$} & $\begin{array}{l}1 \text { Unit } \\
\text { Change }\end{array}$ & 1.007 & $0.980-1.035$ & 0.6080 & 1.000 & $0.971-1.029$ & 0.9904 \\
\hline & Low & 1.000 & & & 1.000 & & \\
\hline & High & 2.107 & $1.155-3.842$ & 0.0150 & 2.354 & $1.235-4.485$ & 0.0092 \\
\hline \multirow{2}{*}{ Race } & Non-white & 1.000 & & & 1.000 & & \\
\hline & White & 1.823 & $0.769-4.321$ & 0.1724 & 1.940 & $0.757-4.973$ & 0.1676 \\
\hline \multirow{2}{*}{$\begin{array}{l}\text { Histologic } \\
\text { type }\end{array}$} & Others & 1.000 & & & 1.000 & & \\
\hline & Ductal & 1.027 & $0.368-2.870$ & 0.9591 & 1.153 & $0.410-3.243$ & 0.7874 \\
\hline \multirow[t]{2}{*}{ Grade } & $1-2$ & 1.000 & & & 1.000 & & \\
\hline & 3 & 2.404 & $1.014-5.698$ & 0.0463 & 2.612 & $1.020-6.688$ & 0.0453 \\
\hline \multirow[t]{2}{*}{$\begin{array}{l}\text { Lymphovascular } \\
\text { invasion }\end{array}$} & No & 1.000 & & & 1.000 & & \\
\hline & Yes & 1.684 & $0.601-4.717$ & 0.3216 & 1.493 & $0.529-4.213$ & 0.4486 \\
\hline \multirow[t]{2}{*}{ Stage } & III & 1.000 & & & 1.000 & & \\
\hline & IV & 4.638 & $1.847-11.647$ & 0.0011 & 5.485 & 2.138-14.069 & 0.0004 \\
\hline \multirow[t]{2}{*}{$\begin{array}{l}\text { Estrogen } \\
\text { receptor }\end{array}$} & No & 1.000 & & & 1.000 & & \\
\hline & Yes & 0.414 & $0.212-0.808$ & 0.0098 & 0.426 & $0.211-0.860$ & 0.0173 \\
\hline \multirow{2}{*}{$\begin{array}{l}\text { Progesterone } \\
\text { receptor }\end{array}$} & No & 1.000 & & & 1.000 & & \\
\hline & Yes & 0.699 & $0.359-1.361$ & 0.2919 & 0.816 & $0.412-1.616$ & 0.5598 \\
\hline \multirow[t]{2}{*}{ HER2 } & No & 1.000 & & & 1.000 & & \\
\hline & Yes & 0.748 & $0.409-1.366$ & 0.3445 & 0.602 & $0.313-1.161$ & 0.1300 \\
\hline \multirow[t]{2}{*}{$\begin{array}{l}\text { Triple-negative } \\
\text { breast cancer }\end{array}$} & No & 1.000 & & & 1.000 & & \\
\hline & Yes & 1.557 & $0.810-2.992$ & 0.1844 & 1.692 & $0.852-3.358$ & 0.1328 \\
\hline \multirow[t]{2}{*}{$\begin{array}{l}\text { Adjuvant } \\
\text { radiation }\end{array}$} & No & 1.000 & & & 1.000 & & \\
\hline & Yes & 0.538 & $0.295-0.982$ & 0.0434 & 0.486 & $0.259-0.914$ & 0.0252 \\
\hline
\end{tabular}

The Kaplan-Meier method was used to evaluate the association of NDRG1 expression and survival over time. Patients with NDRG1-low tumors experienced better actuarial 10-year OS ( $p=0.0129$, Figure $2 a$ ) and DSS ( $p=0.0074$, Figure $2 b$ ). Patients with NDRG1-high tumors showed significantly lower 10-year OS and DSS rates than patients with NDRG1-low (OS, 19\% vs. 45\%, $p=0.0278$; DSS, $22 \%$ vs. $52 \%, p=0.0139$ ). The median OS and DSS times were shorter for NDRG1-high patients (OS, 2.5 years; DSS, 3.1 years) than for NDRG1-low patients (OS, 5.9 years; DSS, 10.7 years). Multivariable model predictors of OS and DSS included NDRG1 expression, ER status, disease stage, and receipt of adjuvant radiation (Table 3). Tumor grade was identified as being associated with OS and DSS at the univariate level but not at the multivariable level. NDRG1-high expression was a strong independent predictor of OS (HR $=2.449,95 \%$ confidence interval $(\mathrm{CI})=1.302-4.607, p=0.0055)$ and DSS $(\mathrm{HR}=$ $2.727,95 \% \mathrm{CI}=1.380-5.389, p=0.0039)$. 
a

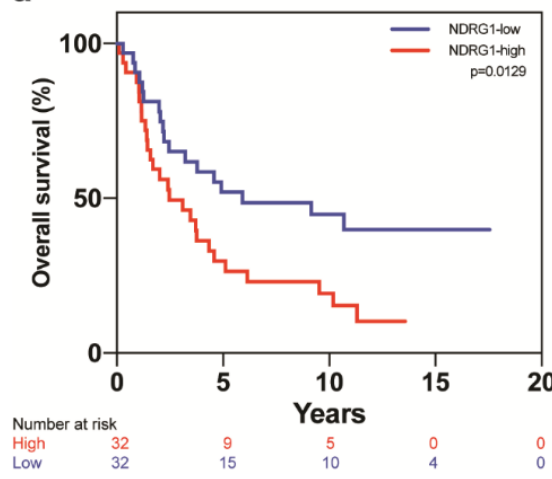

b

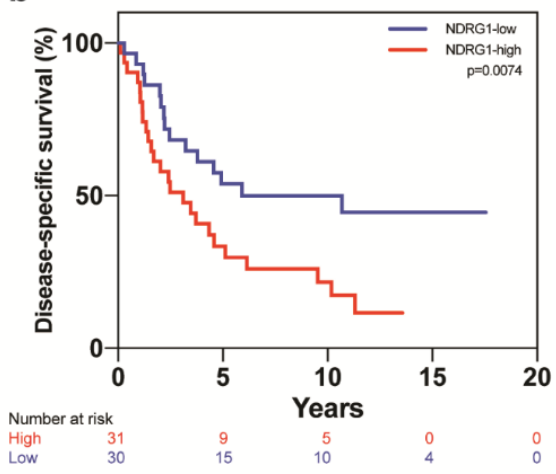

Figure 2. NDRG1 is a predictor of poor outcome in patients with IBC. Kaplan-Meier analysis showed that patients whose tumors had NDRG1-high expression had (a) worse overall survival and (b) worse disease-specific survival than did patients whose tumors had NDRG1-low expression.

Table 3. Multivariate Cox regression analysis on overall survival and disease-specific survival among patients with IBC.

\begin{tabular}{cccccccc}
\hline & & \multicolumn{3}{c}{ Overall Survival } & \multicolumn{3}{c}{ Disease-Specific Survival } \\
\cline { 3 - 7 } Covariate & Level & HR & $\mathbf{9 5 \% ~ C I ~}$ & $p$-Value & HR & $\mathbf{9 5 \% ~ C I ~}$ & $p$-Value \\
\hline NDRG1 & Low & 1.000 & & & 1.000 & & \\
& High & 2.449 & $1.302-4.607$ & 0.0274 & 2.727 & $1.380-5.389$ & 0.0039 \\
Estrogen & No & 1.000 & & & 1.000 & & \\
receptor & Yes & 0.318 & $0.158-0.641$ & 0.0014 & 0.316 & $0.151-0.665$ & 0.0024 \\
& Stage & 1.000 & & & 1.000 & & \\
\multirow{2}{*}{$\begin{array}{l}\text { Adjuvant } \\
\text { radiation }\end{array}$} & IV & 4.350 & $1.685-11.229$ & 0.0024 & 5.070 & $1.908-13.473$ & 0.0011 \\
& No & 1.000 & & & 1.000 & & \\
& Yes & 0.620 & $0.336-1.145$ & 0.1269 & 0.575 & $0.301-1.097$ & 0.0930 \\
\hline
\end{tabular}

ER status was also an important prognostic factor for OS and DSS for patients with IBC; patients with ER-negative tumors had worse OS $(p=0.0077)$ and DSS $(p=0.01)$ relative to patients with ER-positive IBC tumors (Figure 3a,b). Multivariable analysis showed ER status to be an independent factor associated with $\mathrm{OS}(\mathrm{HR}=0.318,95 \% \mathrm{CI}=0.158-0.641, p=0.0014)$ and DSS $(\mathrm{HR}=0.316$, $95 \% \mathrm{CI}=0.151-0.665, p=0.0024$ ) (Table 3). Interestingly, NDRG1-high and ER-negative tumors were associated with the worst clinical outcomes for patients with IBC (OS, $p=0.0003$; DSS, $p=0.0003$; Figure 3c,d). Survival outcomes of ER-positive patients were not affected by NDRG1 expression (Figure 3c,d). Analysis of median OS and DSS times highlights the importance of stratifying patients for both variables: patients with ER-negative tumors had a median of 2.2 years for both OS and DSS, whereas those with ER-negative/NDRG1-high tumors had a median of 1.6 years, and ER-negative/NDRG1-low tumors had medians of 3.2 years OS and 4.6 years DSS (Figure 3e,f).

Disease stage was another independent prognostic variable for OS ( $\mathrm{HR}=4.35,95 \% \mathrm{CI}=1.685-11.229$, $p=0.0024)$ and DSS $(\mathrm{HR}=5.07,95 \% \mathrm{CI}=1.908-13.473, p=0.0024)$. Kaplan-Meyer analysis showed that patients with stage III IBC had better outcomes than did patients with stage IV tumors (OS, $p=0.0003$; DSS, $p<0.0001$ ) (Figure $4 a, b)$. Further stratification of patients with stage III disease according to NDRG1 expression status showed a significant difference in outcomes, wherein patients with stage III NDRG1-high tumors had worse OS $(p=0.045)$ and DSS $(p=0.0239)$ than did patients with stage III NDRG1-low tumors (Figure 4c,d). We could not perform similar analyses of stage IV tumors owing to small patient numbers. Interestingly, the median OS times for patients with stage III tumors differed considerably by NDRG1 expression level, being 9.1 years in NDRG1-low tumors to 4.6 years for 
NDRG1-high stage III tumors (Figure 4e). Similarly, the median DSS times were 4.9 years for stage III NDRG1-low tumors and 10.7 years for stage III NDRG1-high tumors (Figure 4f).

a

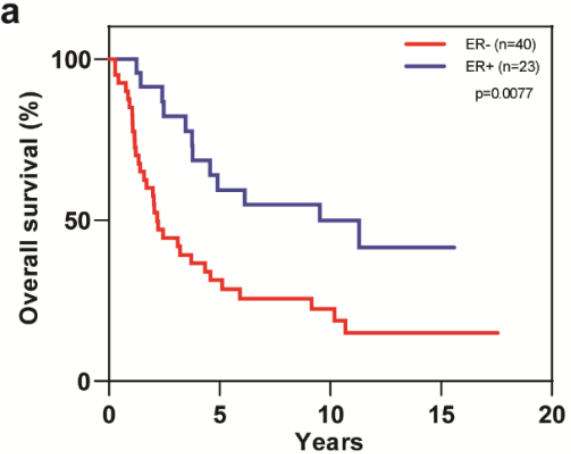

C

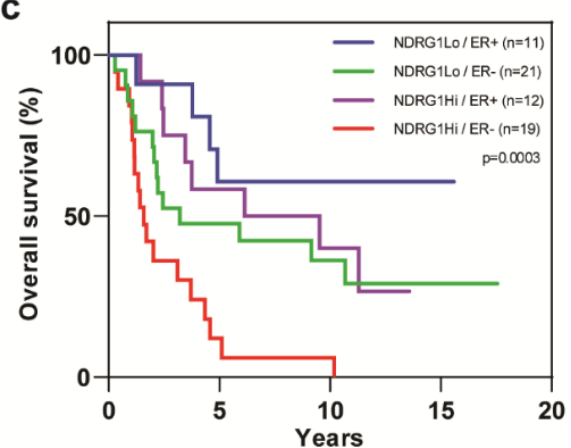

e

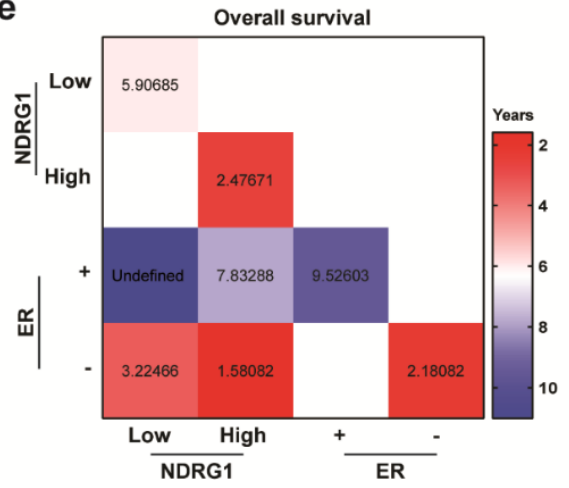

b

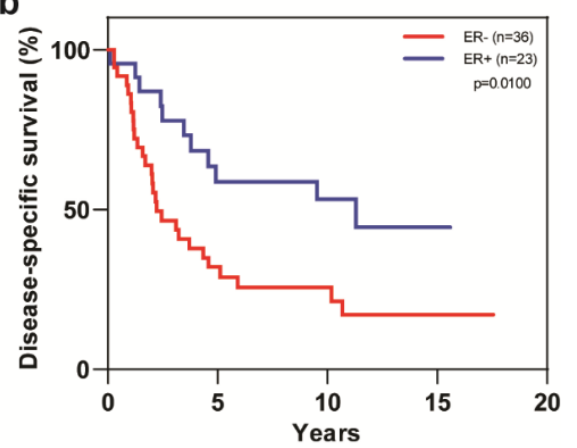

d

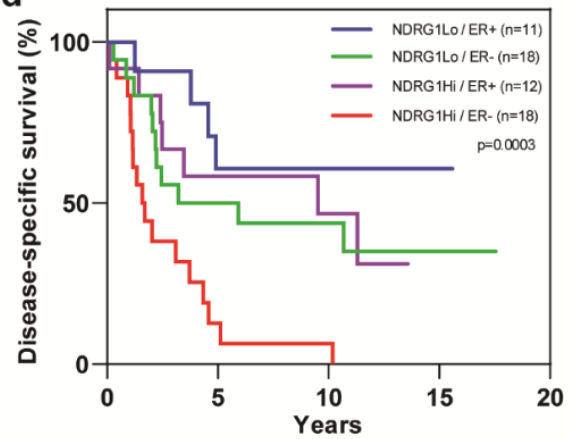

f

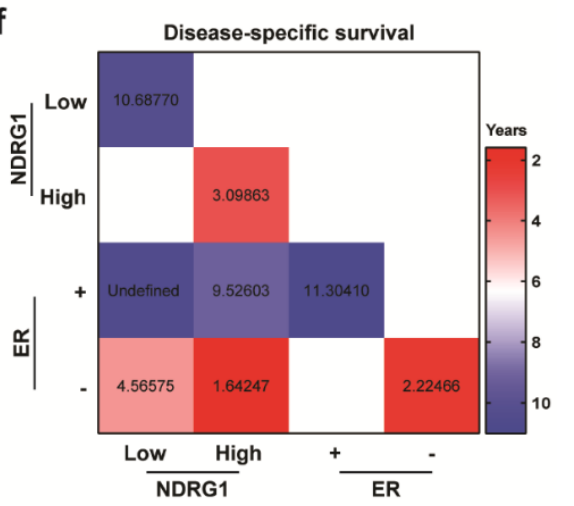

Figure 3. Overall survival and disease-specific survival in patients with IBC stratified by estrogen receptor (ER) status and NDRG1 expression. Patients with ER-negative tumors had (a) worse overall survival and (b) worse disease-specific survival versus patients with ER-positive tumors. (c,d) Stratification of patients by ER and NDRG1 expression status in terms of overall survival and disease-specific survival. Log-rank tests were used to obtain $p$ values. (e,f) Median overall survival and disease-specific survival times, in years, for patients stratified by ER status and NDRG1 expression.

Receipt of adjuvant radiation was also an independent variable marginally related to DSS $(\mathrm{HR}=0.575,95 \% \mathrm{CI}=0.301-1.097, p=0.0930)$ (Table 3$)$. Patients who received adjuvant radiation had better survival outcomes than those who did not (OS, $p=0.0403$; DSS, $p=0.0223$ ) (Figure 5a,b). Among patients who received adjuvant radiation, those with NDRG1-high tumors showed poorer outcomes than those with NDRG1-low tumors (OS, $p=0.0088$; DSS, $p=0.0128$, Figure $5 c, d$ ). Among patients who did not receive adjuvant radiation therapy, NDRG1 expression did not correlate with survival outcomes (Figure 5e,f). The median survival times for all patients who received adjuvant radiation was 3.7 years for OS and 4.6 years for DSS. Stratification of radiation-treated patients by 
NDRG1 again showed distinct differences in survival time, with medians of 3.1 years for both OS and DSS for NDRG1-high tumors versus not achieved for NDRG1-low tumors (Figure 5g,h).

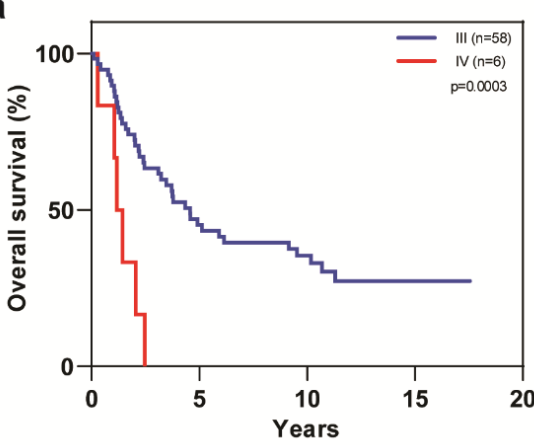

C

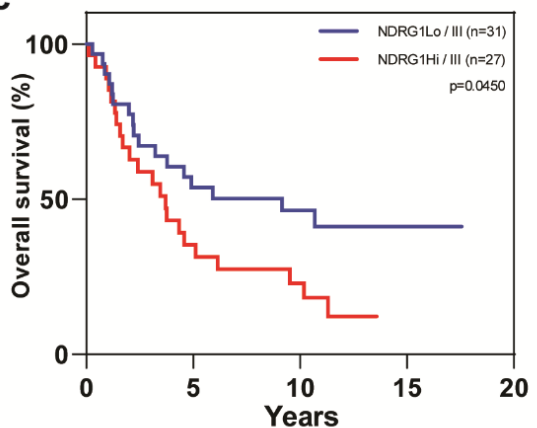

e

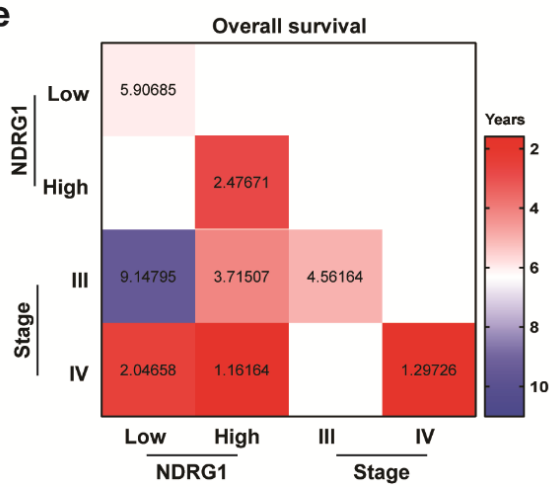

b

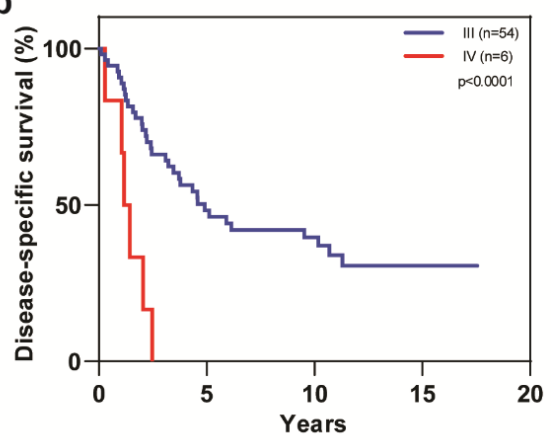

d

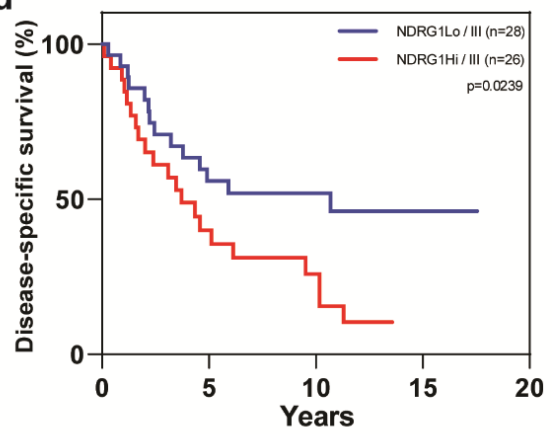

f

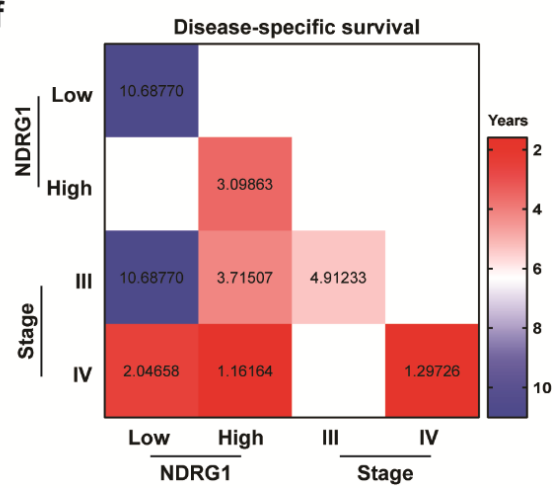

Figure 4. Overall survival and disease-specific survival in patients with IBC stratified by disease stage and NDRG1 expression. Patients with stage III IBC had better (a) overall survival and (b) disease-specific survival than did patients with stage IV IBC. (c,d) Patients with stage III IBC stratified by NDRG1 expression in terms of overall survival and disease-specific survival. Log-rank tests were used to obtain $\mathrm{p}$ values. (e,f) Median overall survival and disease-specific survival times, in years, for patients stratified by disease stage and NDRG1 expression.

As expected, patients with lower tumor grades (I-II) had better outcomes than those with high-grade tumors (OS, $p=0.0399$; DSS, $p=0.0386$; Figure 6a,b). Despite the small number of low-grade tumors, we observed a significant difference in OS $(p=0.0363)$ and DSS $(p=0.0210)$ after stratifying for NDRG1-high versus NDRG1-low expression; patients with low-grade tumors and NDRG1-low expression had better outcomes than patients with NDRG1-high expression (Figure 6c,d). Outcomes may have been worse for patients with high-grade tumors and NDRG1-high expression relative to those with NDRG1-low expression, but those apparent differences were not statistically significant (OS, $p=0.0765$; DSS, $p=0.0699$ ) (Figure 6d,e). Low-grade, NDRG1-high tumors were associated with shorter survival, with median survival times of 4.3 years versus not achieved for low-grade, NDRG1-low tumors (Figure 6g,h). 
A

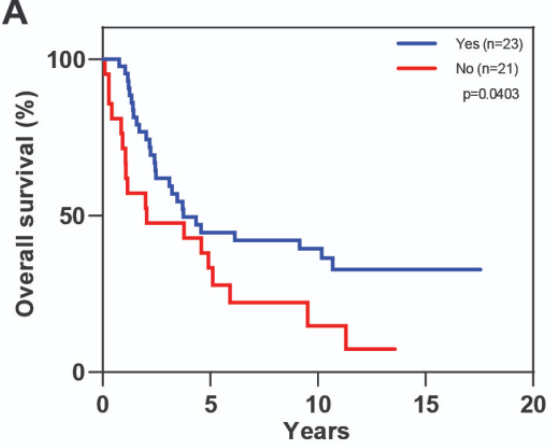

C

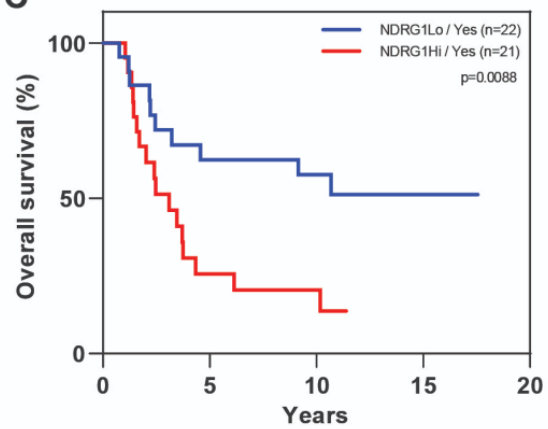

E

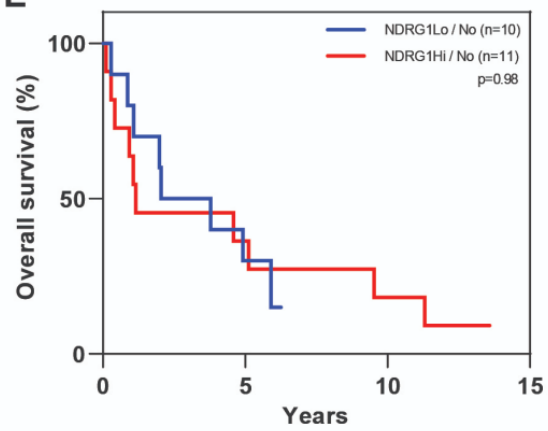

G

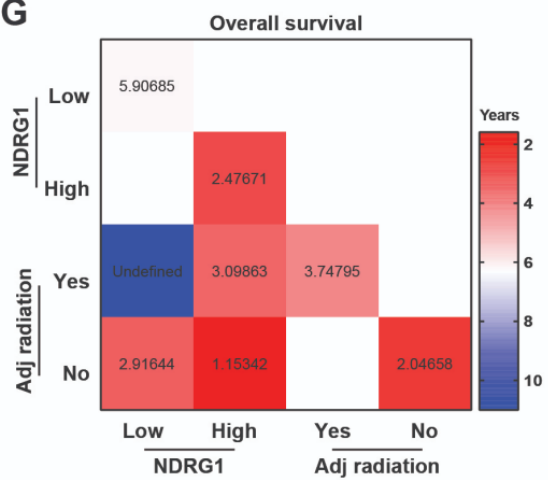

B

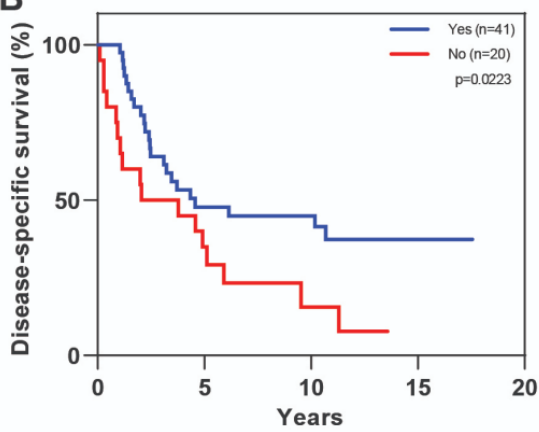

D

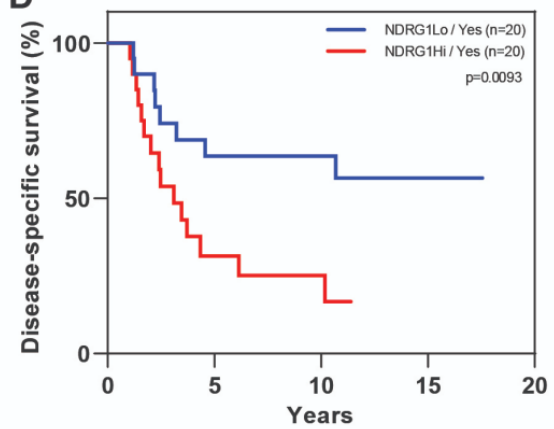

F

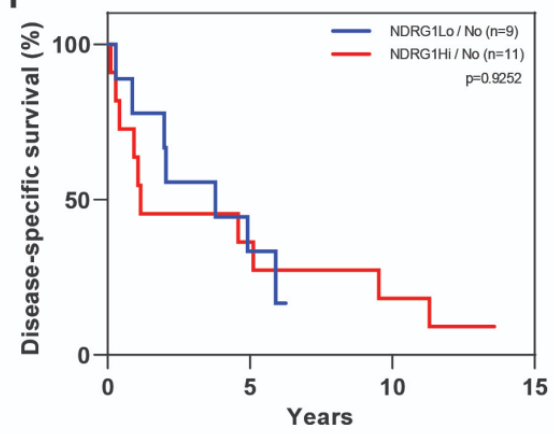

H

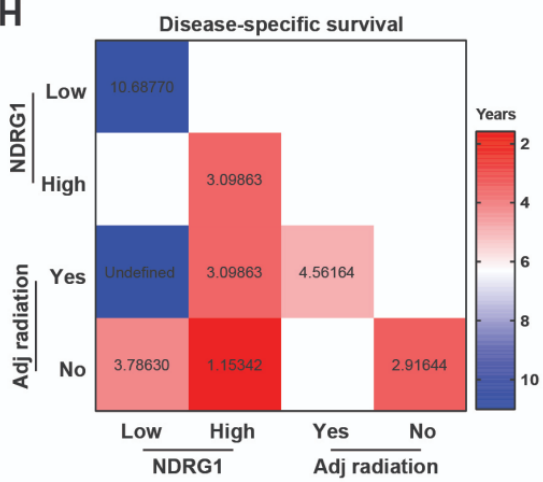

Figure 5. High NDRG1 expression correlated with worse outcomes among patients who received adjuvant radiation therapy. IBC patients who received adjuvant radiation had better (A) overall survival (B) and disease-specific survival than did patients who did not receive radiation. (C,D) Patients who received adjuvant radiation treatment stratified by NDRG1 expression in terms of overall survival and disease-specific survival. (E,F) Patients who did not receive adjuvant radiation stratified by NDRG1 expression in terms of overall survival and disease-specific survival. Log-rank tests were used to obtain $\mathrm{p}$ values. $(\mathbf{G}, \mathbf{H})$ Median overall survival and disease-specific survival times, in years, for patients stratified by NDRG1 expression and adjuvant radiation treatment status. 
a

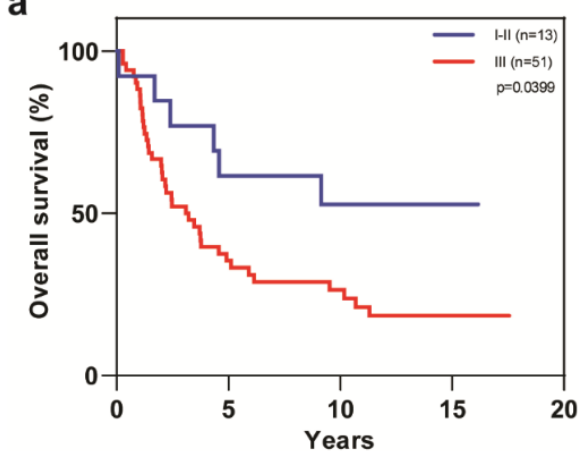

C
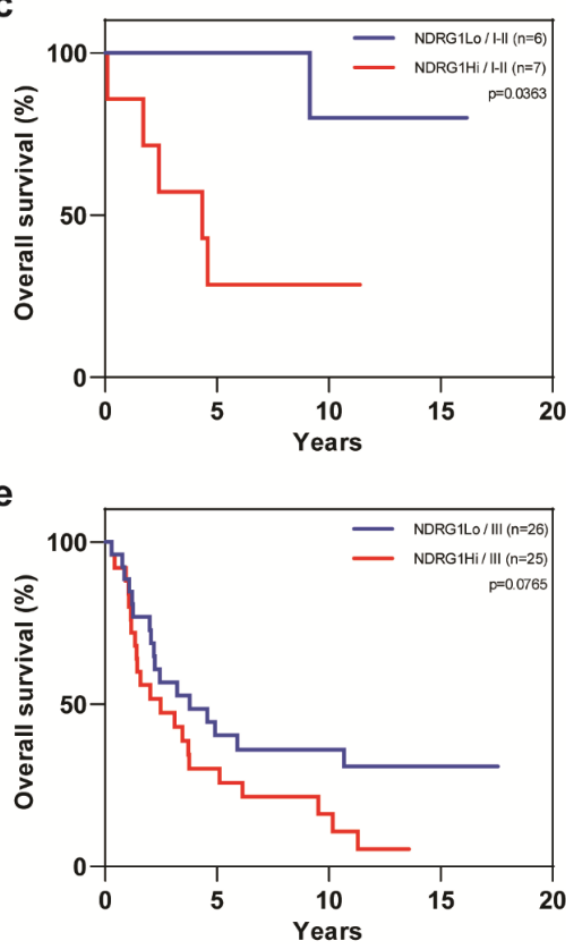

g

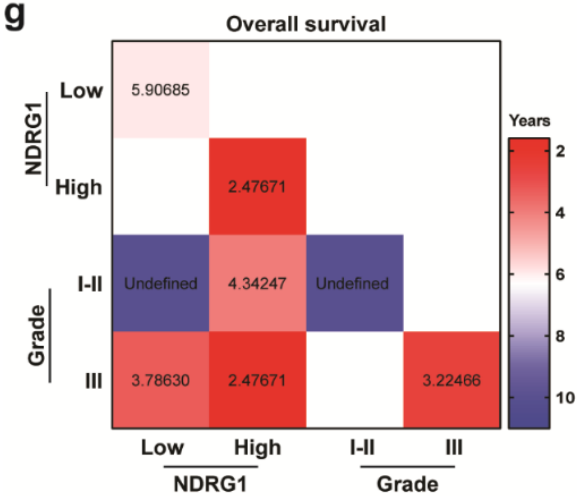

b

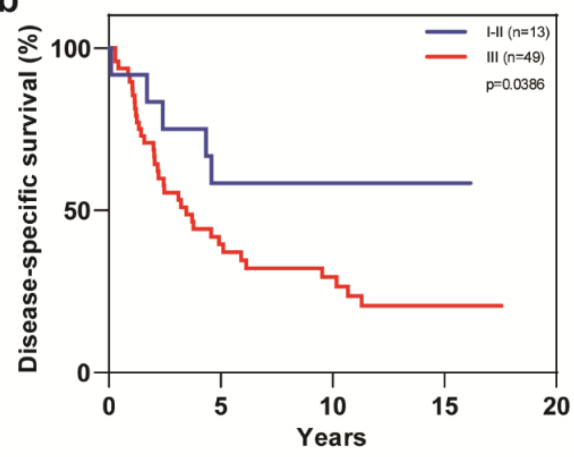

d

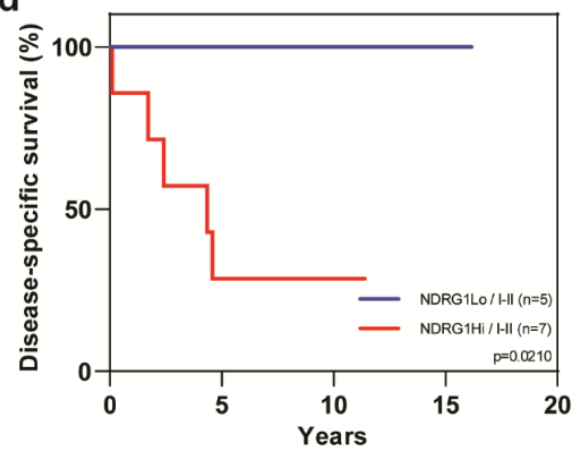

f

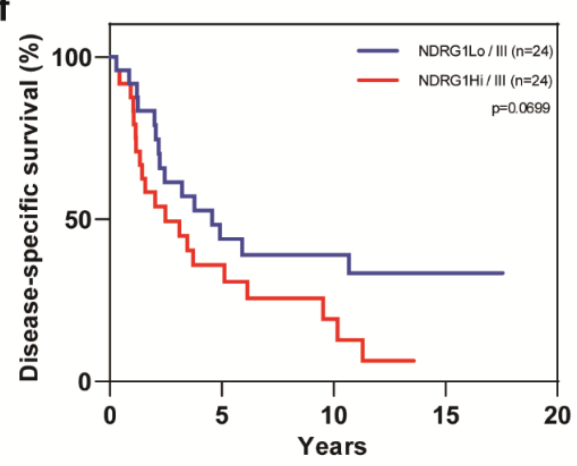

h

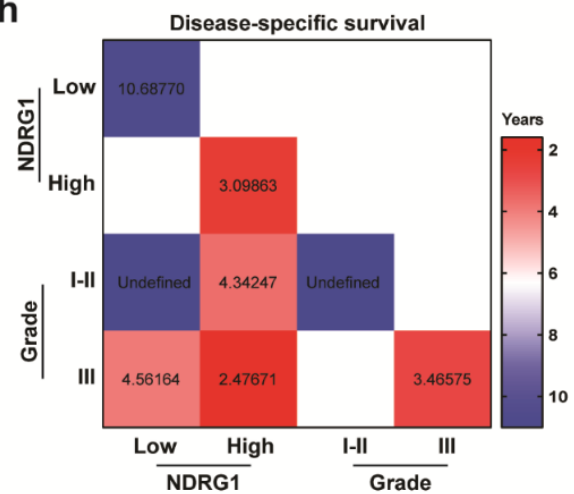

Figure 6. Overall survival and disease-specific survival in patients with IBC stratified by tumor grade and NDRG1 expression. Patients with IBC and low-grade cancer had better (a) overall survival and (b) disease-specific survival than did patients with grade III disease. (c,d) Patients with low-grade IBC stratified by NDRG1 expression in terms of overall survival and disease-specific survival. (e,f) Patients with high-grade IBC stratified by NDRG1 expression in terms of overall survival and disease-specific survival. Log-rank tests were used to obtain $p$ values. (g,h) Median overall survival and disease-specific survival times, in years, for patients stratified by NDRG1 expression and tumor grade. 


\section{Discussion}

IBC remains a relatively poorly defined disease that lacks specific therapeutic targets and prognostic biomarkers; the molecular characterization of IBC could advance our understanding of its unique biology and provide opportunities that could be translated into novel therapeutic strategies to improve clinical outcomes. Herein, we report that NDRG1 protein expression was an independent predictor of poor survival outcomes for patients with IBC. In subset analyses, we report that NDRG1-high expression in patients with ER-negative, stage III tumors and patients who received adjuvant radiation had worse outcomes than did patients with NDRG1-low tumors. Our results suggest that IBC patients could be stratified not only by known prognostic markers but also by biological determinants such as NDRG1 expression status.

NDRG1 is a stress response gene that is highly activated and expressed in hypoxia and resistance to chemotherapy. Its function in breast cancer is widely described as a tumor and metastasis suppressor, acting mainly through inhibition of migration and invasion of cancer cells [20-22,27]. The induction of NDRG1 was shown in a mouse mammary tumor model to suppress metastasis by modulating WNT pathway signaling [21]. Chiang et al. also described how silencing NDRG1 expression in MCF-7 cells led to increased proliferation and invasiveness of those breast cancer cells [27]. Paradoxically, other studies showed that NDRG1 might function as an oncogene or a prognostic biomarker in aggressive forms of breast cancer $[19,23,24,26]$. Mao et al. found that NDRG1 could be used as a marker for invasive breast cancer, observing that NDRG1 expression was significantly higher in invasive breast cancer versus matched non-tumor tissues, and its levels were associated with progression from breast atypia to carcinoma. They also observed a correlation between advanced tumor stage and high NDRG1 expression [23]. Nagai et al. found an association between high NDRG1 expression and worse DSS and OS in a cohort of 600 patients: the 10 year OS rate was $35 \%$ for NDRG1-high versus $67 \%$ for NDRG1-low, and NDRG1 was an independent prognostic factor for both OS and DSS. Moreover, NDRG1 was expressed at higher levels in stage III and IV breast cancer and in grade 3 tumors [26]. More recently, a study by Sevinsky and colleagues observed similar results, wherein analysis of available data sets showed that patients with high expression of NDRG1 had worse recurrence- and metastasis-free survival. Moreover, they demonstrated that NDRG1 promotes breast cancer aggressiveness by altering lipid metabolism [19]. These observations are supported by our ongoing work showing that NDRG1 promotes tumorigenesis and brain metastasis in mouse models of aggressive breast cancer [25].

Expression of the ER is a well-known prognostic and predictive factor, and ER status is essential in the choice of treatment strategy. Patients with ER-positive breast cancer benefit from the use of hormonal therapy and have better OS than do patients with ER-negative disease, and this improvement is independent of disease stage and tumor grade [28-30]. Our results in the present study confirmed that ER status was, indeed, an independent factor related to both OS and DSS, with ER-negative IBC patients exhibiting worse clinical outcomes. ER-negative status is associated with aggressive growth and shorter survival. Interestingly, in our study, stratification of ER-negative patients by NDRG1 expression level showed significant differences in survival outcomes: ER-negative, NDRG1-high tumors were associated with worse outcomes than ER-negative, NDRG1-low tumors. However, no such difference was observed in ER-positive tumors stratified by NDRG1 expression. Our findings indicate that the clinical outcome of patients with ER-negative IBC can be stratified further based on NDRG1 expression status.

Adjuvant radiation therapy is an important part of breast cancer treatment and is known to improve breast cancer-specific survival and reduce tumor recurrence [31-33]. In the current study, we found that receipt of adjuvant radiation for IBC tumors marginally correlated with improved breast cancer-specific survival. We also showed that patients who received adjuvant radiation and had NDRG1 low-expressing tumors had better clinical outcomes than did those with NDRG1-high-expressing radiation-treated tumors. These hypothesis-generating findings suggest that the role of NDRG1 in local failure in breast cancer patients and radiation resistance warrants further investigation. Previous studies of rectal cancer cells have shown that NDRG1 is one of the top highly upregulated genes in 
response to ionizing radiation and that depleting it could be a promising strategy to sensitize cells to radiotherapy [34]. Many studies have been conducted to develop a "radiosensitivity signature" to stratify patients according to benefits from adjuvant radiation treatment [35-37]. However, no such molecular signature for radiation response has yet been identified.

This study has some limitations. First, the IBC TMA comprised tissues from refractory or residual tumors after neoadjuvant systemic therapy. Thus, the expression of NDRG1 described in this cohort may have been influenced by neoadjuvant chemotherapy. Further study that includes patients with pretreated IBC tumors is warranted to further refine these findings. The limited number of patient samples was another limitation of this study. More patient samples are needed to further validate our findings and conduct analyses of some important variables, such as loco-regional recurrence-free survival in patients who received radiation treatment stratified by NDRG1 expression status.

\section{Materials and Methods}

\subsection{IBC Tumor Microarrays and Immunohistochemical Staining}

This study was approved by the institutional review board of The University of Texas MD Anderson Cancer Center (LAB04-0821). Written informed consent was obtained from all patients prior to study enrollment. Details of disease diagnosis, preoperative and postoperative treatments, biomarker studies (including ER, PR, and HER2 status), and TMA construction with post-neoadjuvant residual tumors are reported elsewhere [38]. Briefly, patients received neoadjuvant chemotherapy, which was followed by mastectomy. Patients then received postmastectomy radiation to the chest wall and draining lymphatics (dose ranging from 60 to $71 \mathrm{~Gy}$ ). For hormone receptor status (ER and PR), at least $10 \%$ of invasive cancer cells had to have nuclear staining to be considered positive, analyzed by immunohistochemical staining. HER2 was considered positive if at least $10 \%$ of invasive cancer cells had complete membranous staining in the IHC slide or had positive fluorescence in situ hybridization. For TMA construction, a manual tissue puncher was used to punch three cores of each formalin-fixed and paraffin-embedded post-neoadjuvant residual IBC tumors. Immunohistochemical staining of TMAs was done with a monoclonal antibody against NDRG1 (1:5000, \#9485, Cell Signal) that was previously validated [39]. NDRG1 staining was evaluated by percentage $(0 \%-100 \%)$ and intensity (weak, moderate and strong) of invasive tumor cells showing cytoplasmic and/or membranous staining. NDRG1 H-score was calculated by multiplying the percentage with intensity, and the NDRG1 H-score median (value of 120) was used as a cutoff, wherein 32 patients were grouped as NDRG1-low ( $\leq$ median) and 32 as NDRG1-high (>median). Representative images of NDRG1-low and NDRG1-high tumors are shown in Figure 1. Supplementary file 1 contains the individual data for all variables analyzed and NDRG1 H-score values for each patient.

\subsection{Statistical Analysis}

Patient characteristics were summarized by NDRG1 value (low $[\leq$ median] vs. high $[>$ median]) and compared between patients with NDRG1-low and patients with NDRG1-high tumors. Two-sample t-tests or Wilcoxon rank-sum tests were used for the comparison of continuous variables. Chi-squared tests or Fisher's exact tests were used for the comparison of categorical variables. OS was defined as the interval from diagnosis to death, and DSS as the interval from diagnosis to death from breast cancer. Those patients without an event (death or breast cancer death) were censored at the last follow-up. Kaplan-Meier curves and log-rank tests were used to compare survival distributions. Univariate and multivariate Cox proportional hazards regression models were used to compare OS (and DSS) between NDRG1-low and -high groups, adjusting for other covariates. The proportional hazards assumption was checked by scaled Schoenfeld residual plots and correlation between the scaled Schoenfeld residuals and survival time. P values of $<0.05$ indicated a statistically significant difference. SAS 9.4 (SAS Institute Inc, Cary, NC) was used for data analysis. 


\section{Conclusions}

We are the first to show that NDRG1 expression was an independent prognostic factor for worse survival outcomes in patients with IBC, and together with other important prognostic factors, such as ER status and disease stage, can be used to further stratify prognostic outcome or treatment response in refractory tumors. Our findings suggest that targeting NDRG1 may provide a novel therapeutic strategy to improve outcomes for patients with IBC.

Supplementary Materials: The following are available online at http://www.mdpi.com/2072-6694/12/12/3711/s1, Supplementary file 1: Individual data of the patients for all variables analyzed.

Author Contributions: E.S.V.: conceived and designed the project, performed experiments, analyzed data and interpreted the results, wrote and edited the manuscript with input from all other authors. Y.G.: provided TMA of IBC patient samples, analyzed the TMA and interpreted results. X.H.: performed experiments, analyzed data and interpreted the results. L.H.: analyzed the TMA and interpreted results. E.C.Y.: analyzed the TMA and interpreted results. N.T.U.: provided resources and contributed to the revision of the manuscript. W.A.W.: provided resources and contributed to the revision of the manuscript. D.T.: provided resources and contributed to the revision of the manuscript. J.S.: provided statistical analysis support. B.G.D.: conceived and designed the project, interpreted the results, wrote and edited the manuscript with input from all other authors. All authors have read and agreed to the published version of the manuscript.

Funding: This study was supported in part by the following grants: American Cancer Society Research Scholar grant (RSG-19-126-01 to BGD), Susan G. Komen Career Catalyst Research Grant (CCR16377813 to BGD), and the State of Texas Rare and Aggressive Breast Cancer Research Program.

Acknowledgments: We thank Christine F. Wogan, MS, ELS, of MD Anderson's Division of Radiation Oncology for scientific editing and review of the manuscript, and Carol M. Johnston from the Division of Surgery Histology Core at UT MD Anderson for her help with immunohistochemical staining.

Conflicts of Interest: The authors declare no conflict of interest.

\section{References}

1. Chang, S.; Parker, S.L.; Pham, T.; Buzdar, A.U.; Hursting, S.D. Inflammatory Breast Carcinoma Incidence and Survival. Cancer 1998, 82, 2366-2372. [CrossRef]

2. Hance, K.W.; Anderson, W.F.; Devesa, S.S.; Young, H.A.; Levine, P.H. Trends in inflammatory breast carcinoma incidence and survival: The surveillance, epidemiology, and end results program at the National Cancer Institute. J. Natl. Cancer Inst. 2005, 97, 966-975. [CrossRef] [PubMed]

3. Dirix, L.Y.; Van Dam, P.; Prove, A.; Vermeulen, P.B. Inflammatory breast cancer: Current understanding. Curr. Opin. Oncol. 2006, 18, 563-571. [CrossRef] [PubMed]

4. Wang, Z.; Chen, M.; Pan, J.; Wang, X.; Chen, X.S.; Shen, K.W. Pattern of distant metastases in inflammatory breast cancer-A large-cohort retrospective study. J. Cancer 2020, 11, 292-300. [CrossRef] [PubMed]

5. Cristofanilli, M.; Valero, V.; Buzdar, A.U.; Kau, S.W.; Broglio, K.R.; Gonzalez-Angulo, A.M.; Sneige, N.; Islam, R.; Ueno, N.T.; Buchholz, T.A.; et al. Inflammatory breast cancer (IBC) and patterns of recurrence: Understanding the biology of a unique disease. Cancer 2007, 110, 1436-1444. [CrossRef] [PubMed]

6. Robertson, F.M.; Bondy, M.; Yang, W.; Yamauchi, H.; Wiggins, S.; Kamrudin, S.; Krishnamurthy, S.; Le-Petross, H.; Bidaut, L.; Player, A.N.; et al. Inflammatory breast cancer: The disease, the biology, the treatment. CA Cancer J. Clin. 2010, 60, 351-375. [CrossRef]

7. Fouad, T.M.; Kogawa, T.; Liu, D.D.; Shen, Y.; Masuda, H.; El-Zein, R.; Woodward, W.A.; Chavez-MacGregor, M.; Alvarez, R.H.; Arun, B.; et al. Overall survival differences between patients with inflammatory and noninflammatory breast cancer presenting with distant metastasis at diagnosis. Breast Cancer Res. Treat. 2015, 152, 407-416. [CrossRef]

8. Lim, B.; Woodward, W.A.; Wang, X.; Reuben, J.M.; Ueno, N.T. Inflammatory breast cancer biology: The tumour microenvironment is key. Nat. Rev. Cancer 2018, 18, 485-499. [CrossRef]

9. Zhang, D.; LaFortune, T.A.; Krishnamurthy, S.; Esteva, F.J.; Cristofanilli, M.; Liu, P.; Lucci, A.; Singh, B.; Hung, M.C.; Hortobagyi, G.N.; et al. Epidermal growth factor receptor tyrosine kinase inhibitor reverses mesenchymal to epithelial phenotype and inhibits metastasis in inflammatory breast cancer. Clin. Cancer Res. 2009, 15, 6639-6648. [CrossRef] 
10. Kleer, C.G.; van Golen, K.L.; Braun, T.; Merajver, S.D. Persistent E-cadherin expression in inflammatory breast cancer. Mod. Pathol. 2001, 14, 458-464. [CrossRef]

11. Van Golen, K.L.; Wu, Z.F.; Qiao, X.T.; Bao, L.W.; Merajver, S.D. RhoC GTPase, a novel transforming oncogene for human mammary epithelial cells that partially recapitulates the inflammatory breast cancer phenotype. Cancer Res. 2000, 60, 5832-5838. [PubMed]

12. Costa, R.; Santa-Maria, C.A.; Rossi, G.; Carneiro, B.A.; Chae, Y.K.; Gradishar, W.J.; Giles, F.J.; Cristofanilli, M. Developmental therapeutics for inflammatory breast cancer: Biology and translational directions. Oncotarget 2017, 8, 12417-12432. [CrossRef] [PubMed]

13. Wang, X.; Semba, T.; Phi, L.T.H.; Chainitikun, S.; Iwase, T.; Lim, B.; Ueno, N.T. Targeting Signaling Pathways in Inflammatory Breast Cancer. Cancers 2020, 12, 2479. [CrossRef] [PubMed]

14. Cangul, H. Hypoxia upregulates the expression of the NDRG1 gene leading to its overexpression in various human cancers. BMC Genet. 2004, 5, 27. [CrossRef]

15. Lee, J.C.; Chiang, K.C.; Feng, T.H.; Chen, Y.J.; Chuang, S.T.; Tsui, K.H.; Chung, L.C.; Juang, H.H. The Iron Chelator, Dp44mT, Effectively Inhibits Human Oral Squamous Cell Carcinoma Cell Growth In Vitro and In Vivo. Int. J. Mol. Sci. 2016, 17, 1435. [CrossRef]

16. Fotovati, A.; Abu-Ali, S.; Kage, M.; Shirouzu, K.; Yamana, H.; Kuwano, M. N-myc downstream-regulated gene 1 (NDRG1) a differentiation marker of human breast cancer. Pathol. Oncol. Res. 2011, 17, 525-533. [CrossRef]

17. Chen, Z.; Zhang, D.; Yue, F.; Zheng, M.; Kovacevic, Z.; Richardson, D.R. The iron chelators Dp44mT and DFO inhibit TGF- $\beta$-induced epithelial-mesenchymal transition via up-regulation of N-Myc downstream-regulated gene 1 (NDRG1). J. Biol. Chem. 2012, 287, 17016-17028. [CrossRef]

18. Kovacevic, Z.; Chikhani, S.; Lui, G.Y.; Sivagurunathan, S.; Richardson, D.R. The iron-regulated metastasis suppressor NDRG1 targets NEDD4L, PTEN, and SMAD4 and inhibits the PI3K and Ras signaling pathways. Antioxid. Redox Signal. 2013, 18, 874-887. [CrossRef]

19. Sevinsky, C.J.; Khan, F.; Kokabee, L.; Darehshouri, A.; Maddipati, K.R.; Conklin, D.S. NDRG1 regulates neutral lipid metabolism in breast cancer cells. Breast Cancer Res. 2018, 20, 55. [CrossRef]

20. Bandyopadhyay, S.; Pai, S.K.; Hirota, S.; Hosobe, S.; Takano, Y.; Saito, K.; Piquemal, D.; Commes, T.; Watabe, M.; Gross, S.C.; et al. Role of the putative tumor metastasis suppressor gene Drg-1 in breast cancer progression. Oncogene 2004, 23, 5675-5681. [CrossRef]

21. Liu, W.; Xing, F.; Iiizumi-Gairani, M.; Okuda, H.; Watabe, M.; Pai, S.K.; Pandey, P.R.; Hirota, S.; Kobayashi, A.; Mo, Y.Y.; et al. N-myc downstream regulated gene 1 modulates Wnt- $\beta$-catenin signalling and pleiotropically suppresses metastasis. EMBO Mol. Med. 2012, 4, 93-108. [CrossRef] [PubMed]

22. Godbole, M.; Togar, T.; Patel, K.; Dharavath, B.; Yadav, N.; Janjuha, S.; Gardi, N.; Tiwary, K.; Terwadkar, P.; Desai, S.; et al. Up-regulation of the kinase gene SGK1 by progesterone activates the AP-1-NDRG1 axis in both PR-positive and -negative breast cancer cells. J. Biol. Chem. 2018, 293, 19263-19276. [CrossRef] [PubMed]

23. Mao, X.Y.; Fan, C.F.; Wei, J.; Liu, C.; Zheng, H.C.; Yao, F.; Jin, F. Increased N-myc downstream-regulated gene 1 expression is associated with breast atypia-to-carcinoma progression. Tumour Biol. 2011, 32, 1271-1276. [CrossRef] [PubMed]

24. Li, E.Y.; Huang, W.Y.; Chang, Y.C.; Tsai, M.H.; Chuang, E.Y.; Kuok, Q.Y.; Bai, S.T.; Chao, L.Y.; Sher, Y.P.; Lai, L.C. Aryl Hydrocarbon Receptor Activates NDRG1 Transcription under Hypoxia in Breast Cancer Cells. Sci. Rep. 2016, 6, 20808. [CrossRef] [PubMed]

25. Villodre, E.S.; Hu, X.; Larson, R.; Eckhardt, B.L.; Gong, Y.; Huo, L.; Song, J.; Krishnamurthy, S.; Ibrahim, N.K.; Ueno, N.T.; et al. Abstract P3-01-10: Ndrg1-egfr axis in inflammatory breast cancer tumorigenesis and brain metastasis. Cancer Res. 2020, 80, P3-01-10. [CrossRef]

26. Nagai, M.A.; Gerhard, R.; Fregnani, J.H.; Nonogaki, S.; Rierger, R.B.; Netto, M.M.; Soares, F.A. Prognostic value of NDRG1 and SPARC protein expression in breast cancer patients. Breast Cancer Res. Treat. 2011, 126, 1-14. [CrossRef]

27. Chiang, K.C.; Yeh, C.N.; Chung, L.C.; Feng, T.H.; Sun, C.C.; Chen, M.F.; Jan, Y.Y.; Yeh, T.S.; Chen, S.C.; Juang, H.H. WNT-1 inducible signaling pathway protein-1 enhances growth and tumorigenesis in human breast cancer. Sci. Rep. 2015, 5, 8686. [CrossRef]

28. Yip, C.H.; Rhodes, A. Estrogen and progesterone receptors in breast cancer. Future Oncol. 2014, 10, $2293-2301$. [CrossRef] 
29. Grann, V.R.; Troxel, A.B.; Zojwalla, N.J.; Jacobson, J.S.; Hershman, D.; Neugut, A.I. Hormone receptor status and survival in a population-based cohort of patients with breast carcinoma. Cancer 2005, 103, 2241-2251. [CrossRef]

30. Munoz, D.F.; Plevritis, S.K. Estimating Breast Cancer Survival by Molecular Subtype in the Absence of Screening and Adjuvant Treatment. Med. Decis. Mak. 2018, 38, 32S-43S. [CrossRef]

31. Daugherty, E.C.; Daugherty, M.R.; Bogart, J.A.; Shapiro, A. Adjuvant Radiation Improves Survival in Older Women Following Breast-Conserving Surgery for Estrogen Receptor-Negative Breast Cancer. Clin. Breast Cancer 2016, 16, 500-506. [CrossRef] [PubMed]

32. Speers, C.; Pierce, L.J. Postoperative Radiotherapy After Breast-Conserving Surgery for Early-Stage Breast Cancer: A Review. JAMA Oncol. 2016, 2, 1075-1082. [CrossRef] [PubMed]

33. Ohri, N.; Haffty, B.G. The evolution of adjuvant radiation therapy for early-stage and locally advanced breast cancer. Breast J. 2020, 26, 59-64. [CrossRef] [PubMed]

34. Kim, S.C.; Shin, Y.K.; Kim, Y.A.; Jang, S.G.; Ku, J.L. Identification of genes inducing resistance to ionizing radiation in human rectal cancer cell lines: Re-sensitization of radio-resistant rectal cancer cells through down regulating NDRG1. BMC Cancer 2018, 18, 594. [CrossRef] [PubMed]

35. Servant, N.; Bollet, M.A.; Halfwerk, H.; Bleakley, K.; Kreike, B.; Jacob, L.; Sie, D.; Kerkhoven, R.M.; Hupe, P.; Hadhri, R.; et al. Search for a gene expression signature of breast cancer local recurrence in young women. Clin. Cancer Res. 2012, 18, 1704-1715. [CrossRef] [PubMed]

36. Tramm, T.; Mohammed, H.; Myhre, S.; Kyndi, M.; Alsner, J.; Borresen-Dale, A.L.; Sorlie, T.; Frigessi, A.; Overgaard, J. Development and validation of a gene profile predicting benefit of postmastectomy radiotherapy in patients with high-risk breast cancer: A study of gene expression in the DBCG82bc cohort. Clin. Cancer Res. 2014, 20, 5272-5280. [CrossRef]

37. Speers, C.; Zhao, S.; Liu, M.; Bartelink, H.; Pierce, L.J.; Feng, F.Y. Development and Validation of a Novel Radiosensitivity Signature in Human Breast Cancer. Clin. Cancer Res. 2015, 21, 3667-3677. [CrossRef]

38. Gong, Y.; Huo, L.; Liu, P.; Sneige, N.; Sun, X.; Ueno, N.T.; Lucci, A.; Buchholz, T.A.; Valero, V.; Cristofanilli, M. Polycomb group protein EZH2 is frequently expressed in inflammatory breast cancer and is predictive of worse clinical outcome. Cancer 2011, 117, 5476-5484. [CrossRef]

39. Zeng, L.; Deng, X.; Zhong, J.; Yuan, L.; Tao, X.; Zhang, S.; Zeng, Y.; He, G.; Tan, P.; Tao, Y. Prognostic value of biomarkers EpCAM and $\alpha \mathrm{B}$-crystallin associated with lymphatic metastasis in breast cancer by iTRAQ analysis. BMC Cancer 2019, 19, 831. [CrossRef]

Publisher's Note: MDPI stays neutral with regard to jurisdictional claims in published maps and institutional affiliations.

(C) 2020 by the authors. Licensee MDPI, Basel, Switzerland. This article is an open access article distributed under the terms and conditions of the Creative Commons Attribution (CC BY) license (http://creativecommons.org/licenses/by/4.0/). 